EPJ Web of Conferences 31, 00038 (2012)

DOI: $10.1051 /$ epjconf/20123100038

(C) Owned by the authors, published by EDP Sciences - SIF, 2012

\title{
Statistical (?) decay of light hot nuclei
}

\author{
G. Baiocco ${ }^{1,2}$, M. Bruno ${ }^{1}$, M. D'Agostino ${ }^{1}$, L. Morelli $^{1}$, \\ (NUCL-EX Collaboration ${ }^{3,4,5}$ ) \\ F. Gulminelli ${ }^{2}$ and AD. R. RAduta ${ }^{6}$
}

${ }^{1}$ Dipartimento di Fisica dell'Università and INFN, Bologna, Italy

${ }^{2}$ CNRS, UMR6534, LPC, F-14050 Caen cédex, France and

ENSICAEN, UMR6534, LPC, F-14050 Caen cédex, France

${ }^{3}$ INFN, Firenze, Italy

${ }^{4}$ INFN, Laboratori Nazionali di Legnaro, Italy

${ }^{5}$ Dipartimento di Fisica dell'Università, Padova, Italy

${ }^{6}$ NIPNE, Bucharest-Măgurele, POB-MG6, Romania

\begin{abstract}
The reaction ${ }^{12} \mathrm{C}+{ }^{12} \mathrm{C}$ at $95 \mathrm{MeV}$ beam energy has been measured using the GARFIELD+RCo apparatuses at Laboratori Nazionali di Legnaro LNL - INFN, Italy, in the framework of an experimental campaign proposed by the NUCL-EX collaboration. The aim is to progress in the understanding of statistical properties of light nuclei at excitation energies above particle emission thresholds, by measuring exclusive fusion-evaporation data. A theoretical study of the system, performed with a newly developed Monte Carlo Hauser-Feshbach code, is shown, together with preliminary results of the data analysis.
\end{abstract}

\section{Introduction}

Dissipative nuclear reactions are a tool to investigate nuclear properties at finite temperature, notably including the excitation energy dependence of the nucleon effective mass, symmetry energy and pairing correlations. A general concern is associated with such experimental studies: the final inclusive

This is an Open Access article distributed under the terms of the Creative Commons Attribution License 2.0, which permits unrestricted use, distribution, and reproduction in any medium, provided the original work is properly cited. 
yields represent integrated contributions and, because of that, the information they bear on specific excitation energy (temperature) regions of the nuclei explored during the reaction may be model dependent. The challenge is therefore to perform a highly exclusive detection of reaction products, in order to backtrace their origin, with the aim of constraining nuclear properties at finite temperature [1]. An additional challenge comes into play in modelization: because of the strong interplay of nuclear structure and reactions, statistical (and dynamical) codes should be highly constrained by available nuclear data on ground state properties and information on low excitation energies, with the aim of gaining a better predictive power on finite temperature observables.

In this work, exclusive data for the reaction ${ }^{12} \mathrm{C}+{ }^{12} \mathrm{C}$ at $95 \mathrm{MeV}$, measured at LNL using the GARFIELD+RCo [2] apparatuses, are compared to the predictions of a Monte Carlo Hauser-Feshbach (HF) code, explicitly including all the experimentally measured particle unstable levels from the archive NUDAT2 (http://www.nndc.bnl.gov/nudat2/). Main physics goals are the determination of the nuclear level density in the $A \sim 20$ region, the understanding of the statistical behavior of light nuclei with excitation energies $\sim 3 \mathrm{~A} . \mathrm{MeV}$, and the measurement of observables linked to the presence of cluster structures of excited levels.

\section{The statistical decay code}

A full description of the statistical decay code is given in [3]. A special attention has been paid to the choice of a realistic parameterization for the nuclear Level Density (LD). In [4] the parameters of the nuclear LD for the Backshifted Fermi Gas model (BSFG) have been determined for a large set of nuclei, by the fit of complete level schemes at low excitation energies. This semi-empirical model perfectly matches our requests, because of the possibility to fit the low energy spectroscopic information with the same functional form, adequate to the description of the high energy behavior. Moreover, reliable extrapolation formulas are proposed for the LD parameters of light nuclei not included in the fitted data set. Parameters obtained in [4] are affected by structure effects (pairing and shell effects), and reliable up to $e^{*} \sim 1 \mathrm{~A} . \mathrm{MeV}$ for $A \sim 20$ nuclei. Higher energy constraints come from evaporation studies, at $e^{*} \sim 3 \mathrm{~A} . \mathrm{MeV}[5]$, and the transition between these two energy regimes can be ascribed to the variation of the LD parameter $a$ with the excitation energy. We have therefore adopted a matched $a_{M}\left(E^{*}\right)$ in the BSFG model of [4], whose functional form reproduces the transition 


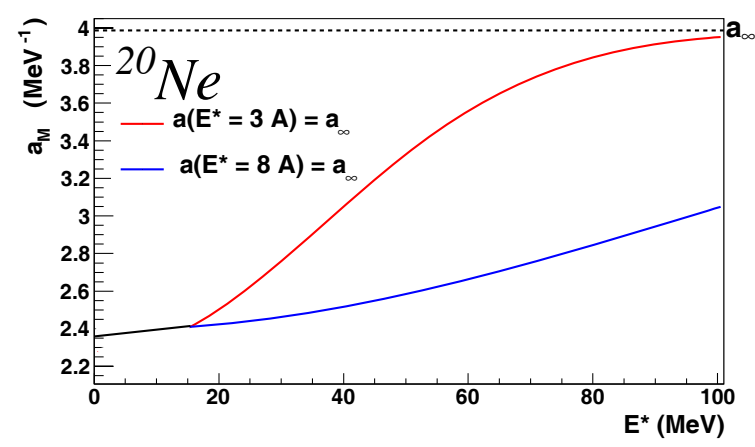

Figure 1: (Color online) Functional form of $a_{M}\left(E^{*}\right)$ implemented in our HF code for ${ }^{20} \mathrm{Ne}$ : the black line corresponds to the constraint given in [4], the asymptotic $a_{\infty}$ is taken from [5], and the blue and red curve correspond to two different choices of the parameter governing the rapidity of the increase.

from the low energy constraints given in [4] to an asymptotic value coming from [5]. The rapidity of the variation has to be determined through comparison to data. As an example, results for two different choices of the parameter governing this rapidity are shown in fig. 1 for ${ }^{20} \mathrm{Ne}$.

\section{Experimental observables and calculations}

The compound nucleus issued in case of complete fusion for the reaction ${ }^{12} \mathrm{C}+{ }^{12} \mathrm{C}$ at $95 \mathrm{MeV}$ beam energy is ${ }^{24} \mathrm{Mg}$, at $e^{*}=2.6 \mathrm{~A} . \mathrm{MeV}$ and with even values of the initial angular momentum $J_{0}$, extracted from a triangular distribution with $J_{0 \max }=12 \hbar$.

At present, due to the in progress status of energy calibrations, the fusion-evaporation channel is selected thanks to static conditions on the total detected charge ( $\geq 80 \%$ of the charge available in the entrance channel) and on the coincidence between a residue at forward angles (RCo) and light particles detected in GARFIELD $\left(\theta_{l a b} \geq 30^{\circ}\right)$. The charge distribution corresponding to this preliminary selection is shown in fig.2, together with the energy spectra of protons and $\alpha$ detected at GARFIELD angles. Code calculations, reported in the figure for two possible values of the LD model free parameter, show that the shape of the charge distribution is globally well reproduced, in particular concerning the trend of the observed odd-even effects. A very good reproduction of the proton energy spectrum is achieved, while the same choice for the LD cannot reproduce the energy spectrum of 

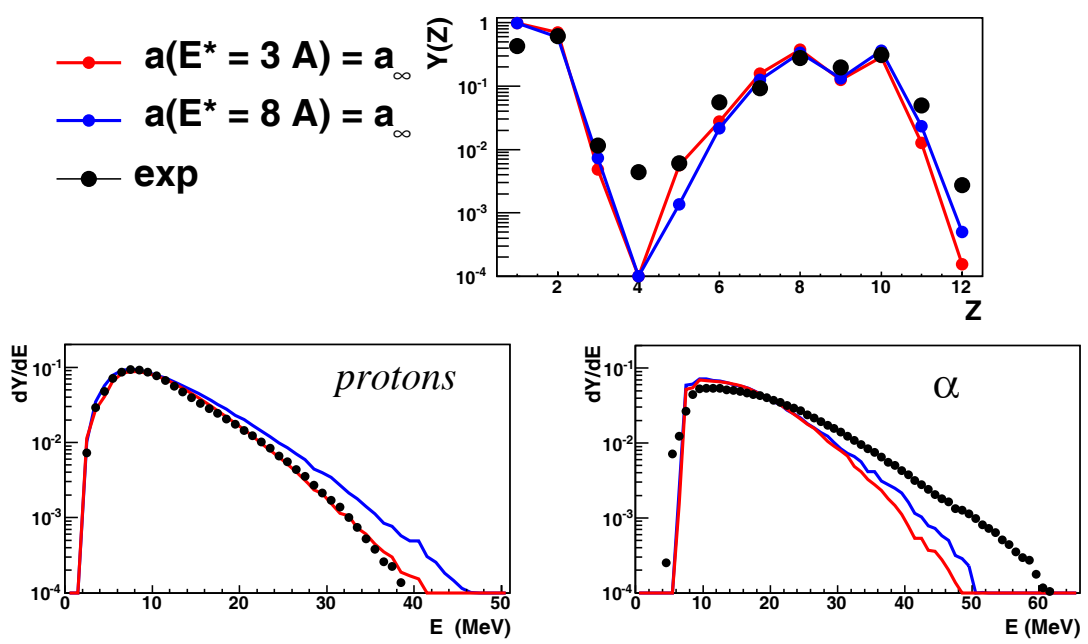

Figure 2: (Color online) Experimental data (black dots) and HF calculations (red and blue dots and lines, same color scheme of fig.1) for ${ }^{12} \mathrm{C}+{ }^{12} \mathrm{C}$, under a preliminary selection of the fusion-evaporation channel. Upper panel: charge distribution. Lower panel, left: proton energy spectrum; right: $\alpha$ energy spectrum.

$\alpha$ particles. This deviation from a statistical behavior can be attributed to pre-equilibrium effects. Having highly constrained the code via experimental information, these effects are tentatively interpreted as $\alpha$-clustering effects, because of the even-even $N=Z$ nature of the ${ }^{12} \mathrm{C}$ projectile and target.

\section{References}

[1] D’Agostino M. et al., Nucl. Phys. A 875 (2012) 139, and contribution to this conference.

[2] Gramegna F. et al., Nucl. Instr. and Meth. A 389 (1997) 474;

Moroni A. et al., Nucl. Instr. and Meth. A 556 (2006) 516.

[3] Baiocco G., PhD Thesis, University of Bologna and University of CaenLower Normandy (2012), http://tel.archives-ouvertes.fr/lab/lpcc/ .

[4] Von Egidy T. and Bucurescu D., Phys. Rev. C 72 (2005) 044311.

[5] Töke J. and Swiatecki W. J., Nucl. Phys. A 372 (1981) 141. 